\title{
Bragg-Scattering Four-Wave Mixing in Nonlinear Fibers with Intracavity Frequency-Shifted Laser Pumps
}

\author{
Katarzyna Krupa, ${ }^{1}$ Michela Bettenzana, ${ }^{2}$ Alessandro Tonello, ${ }^{1}$ Vincent Couderc, ${ }^{1}$ \\ Philippe Di Bin, ${ }^{1}$ Stefan Wabnitz, ${ }^{2}$ and Alain Barthélémy ${ }^{1}$ \\ ${ }^{1}$ XLIM, UMR CNRS 6172, Université de Limoges, 123 av A. Thomas, 87060 Limoges, France \\ ${ }^{2}$ Dipartimento di Ingegneria dell'Informazione, Università di Brescia, Via Branze 38, 25123 Brescia, Italy
}

Correspondence should be addressed to Alessandro Tonello, alessandro.tonello@unilim.fr

Received 8 July 2011; Accepted 5 September 2011

Academic Editor: Christophe Finot

Copyright ( 2012 Katarzyna Krupa et al. This is an open access article distributed under the Creative Commons Attribution License, which permits unrestricted use, distribution, and reproduction in any medium, provided the original work is properly cited.

We experimentally study four-wave mixing in highly nonlinear fibers using two independent and partially coherent laser pumps and a third coherent signal. We focus our attention on the Bragg-scattering frequency conversion. The two pumps were obtained by amplifying two Intracavity frequency-shifted feedback lasers working in a continuous wave regime.

\section{Introduction}

Over the last decades, the nonlinear phenomena of parametric four-wave mixing (FWM) have been intensively studied in view of their great potential to provide a large variety of all-optical functionalities for ultrafast signal processing [14]. In particular, optical parametric amplification, frequency conversion, phase conjugation, and nonlinear switching have been widely explored. The main interest was developed for FWM with coherent pumps, since this technique may provide high conversion efficiencies with appropriate dispersion curves [5]. Nevertheless, in recent years, FWM in optical fibers with incoherent or partially coherent pumps has attracted great attention, in view of its polarizationindependent gain and increased resilience to stimulated Brillouin scattering (SBS) $[6,7]$. Let us remember that SBS limits the direct use of intense and narrowband lasers in nonlinear fiber. For the specific case of highly nonlinear fibers (hereafter HNLF) the Brillouin threshold may be in the order of few tens of milliwatt for a fiber length of $500 \mathrm{~m}$. The common way to increase the Brillouin threshold requires the adoption of phase modulation of pumps. For the case of two-pump parametric devices, optimal architectures of phase modulations have been proposed to suppress SBS. These solutions are based on phase modulators, generally driven by a pseudorandom bitsequence generator $[4,5,8,9]$ at $\mathrm{GHz}$ repetition frequency. With pure phase modulation the pumps can be spectrally broadened without affecting the time domain intensity.

The adoption of incoherent pumps may represent a costeffective solution to study FWM in different scenarios and may open a novel kind of applications, even if this may take place at the expense of low conversion efficiency and spectral broadening of the converted signal. The interplay between four-wave mixing processes with mixed coherent-incoherent pumps may also open entirely new features as reported very recently in [10]. Till now, most of investigations on nonlinear effects with incoherent pumping have been conducted for single pump FWM processes and so far, little attention has been paid to a specific type of FWM generally called Braggscattering FWM (BS-FWM) [6, 10]. The low-noise nature of the BS-FWM is a very attractive feature $[8,11]$, which makes this process an excellent candidate for light-by-light manipulation with possible extension to the case of very weak signals. These optical functionalities may be of interest to manipulate optical quantum states in view of frequency conversion in quantum networks.

In this paper, we study the case of BS-FWM with partially coherent pumps in HNLF. For this purpose we used two broadband intra-cavity frequency-shifted feedback lasers 
(IFSFLs) that operated in the continuous wave (CW) regime. Although these pumps are independent and exhibit low coherence time, we experimentally observed a clear signature of frequency conversion. In addition, we discuss the competition between BS-FWM and other types of FWM simultaneously occurring in HNLFs. In particular, we experimentally demonstrate the competition with degenerate (i.e., single pump) FWM.

\section{Experimental Setup and Pump Sources}

In the case of a parametric frequency conversion, the coherence time of the pump $t_{C}$ should be compared to the characteristic evolution time $t_{P}$ to establish such a nonlinear interaction. In HNLF and for powers in the order of $50 \mathrm{~mW}$ one could estimate $t_{P}$ from the effective nonlinear length and group speed [12]. Whereas $t_{C}$ from our pumps is instead associated to the bandwidth of at least $50 \mathrm{GHz}$ of our pumps, so we may expect that the nonlinear interplay becomes affected by the partial coherence of pumps.

To study the case of BS-FWM frequency conversion in such a different regime we propose the experimental setup presented in Figure 1. Instead of filtering amplified spontaneous emission (ASE) sources (often employed in previous studies of incoherent FWM [6]) in our experiment we used a pair of intra-cavity frequency-shifted feedback lasers (IFSFLs) that we independently amplified to obtain two intense pumps.

The coherence properties of IFSFLs have been widely investigated and exploited in metrology and in astronomical imaging [13-15]. This kind of laser has also been proposed to increase by several orders of magnitude the SBS threshold in [16] allowing supercontinuum generation from an amplified IFSFL with several Watts of input power. However BSFWM requires two distinct pumps. Thus, we developed two identical tunable fiber IFSFLs that operated at different wavelengths. Each IFSFL has been separately amplified by an erbium-doped fiber amplifier (EDFA) and protected against unwanted back reflections by an isolator. To reduce the ASE noise we put one bandpass filter at the input and two bandpass filters at the output of each EDFA (DiCon thin-film filters with $5 \mathrm{~nm}$ bandwidth each). These sets of filters carve the spectrum of each pump to reduce the background spectral level without significantly affecting the $3 \mathrm{~dB}$ bandwidth of the sources. Next, the two pumps were combined by a 50/50 fiber coupler and multiplexed with a third signal from a commercially available tunable extended cavity laser (laser power of $4 \mathrm{~mW}$ ) through a 95/5 "tap" coupler. Pumps and signal were injected into a $480 \mathrm{~m}$ long off-the-shelf segment of HNLF with nominal zero dispersion wavelength (ZDW) of $1545 \mathrm{~nm}$.

The spectrum at the output end of the fiber was analyzed by an optical spectrum analyzer (OSA) and by a $1 \mathrm{GHz}$ photodiode connected either to an oscilloscope (OSC) or to a radio frequency $(\mathrm{RF})$ analyzer. In what follows we will refer to a set of measurements in which the average powers of pump 1, 2, and signal at the input end of HNLF were $43 \mathrm{~mW}$, $62 \mathrm{~mW}$, and $0.12 \mathrm{~mW}$, respectively.
The two central elements of our experimental setup are the fiber IFSFLs. We present their synoptic scheme in Figure 2. Each laser cavity consists of a $20 \mathrm{~m}$ long polarization maintaining (PM) erbium-doped fiber pumped by a laser diode emitting at $1480 \mathrm{~nm}$ and an inline PM acoustooptic frequency shifter (AOFS) controlled by a fixed RF driver at $110 \mathrm{MHz}$. The cavity mirrors are provided by a fiber FC-PC connector (see Figure 2, on the right) to introduce a natural Fresnel-type back reflection; the laser is connected to an output fiber with a FC-APC connector though a mating sleeve containing an air gap. The second mirror is represented on the left-hand side of Figure 2 by a blazed grating in Littrow configuration working at the first diffraction order. The microlens has a focal length $f^{\prime}=8 \mathrm{~mm}$ while the grating has density of 600 grooves per millimeter. The central wavelength of the laser can be freely tuned all over the bandwidth permitted by the erbium gain by a simple rotation of the grating. The two IFSFLs are identical copies, and they are both based on this scheme: they were tuned to the upper and lower spectrum edge allowed by such lasers (1540 $\mathrm{nm}$ and $1563 \mathrm{~nm}$ for pump 1 and 2, resp.) so as to obtain the largest possible difference between their carrier frequencies. The corresponding frequency difference in this case is of $2.87 \mathrm{THz}$.

The free-space optics section reported in Figure 2 provides the transfer function of intra-cavity frequency dependent loss. In the case of perfect collimation, it turns out that the filtered bandwidth is inversely proportional to the focal length $f^{\prime}$ of the microlens. The fiber end close to the lens was cleaved with an angle of $8^{\circ}$ to reduce any undesired back reflection. Under these conditions the low numerical aperture of the fiber provides the filtering mechanism. In order to smooth the optical spectrum, we used the AOFS inside the fiber cavity. A dynamical equilibrium can be found between frequency shifting, tight frequency-dependent loss, and broadband gain. However, a more detailed analysis of the RF spectrum at the output of one of the IFSFL unveils its complex behavior, as reported on the left-hand side of Figure 3.

We observe the presence of a comb of frequencies regularly spaced by $3.6 \mathrm{MHz}$, which compares well with the reciprocal of the round trip time of the fiber cavity. We compare these results with the direct numerical calculation coming from a model described in [17] (see Figure 3(b)). To implement equation (3) of [17] we assumed a fiber length of $27 \mathrm{~m}$ (in fact both the doped fiber and the AOFS are spliced to PM standard monomode fiber patchcords on both ends), a group index of 1.45 , a frequency shift of $2 \times 110 \mathrm{MHz}$ (the AOFS is seen two times per round trip), and a random phase for each frequency comb component. Note that in our case the AOFS is driven by a radio frequency which is much higher than the inverse of the round trip time of the cavity.

The dynamical evolution of the IFSFLs is also a key point for applications in nonlinear optics. Indeed we observed as many other authors that our IFSFLs may operate in a CW or in a pulsed regime (see, e.g., [18]), depending on the level of feedback in the cavity. However, it would be desirable that the IFSFLs work close to a CW mode to observe BS-FWM as one would expect that a nonsynchronized pulsed regime 


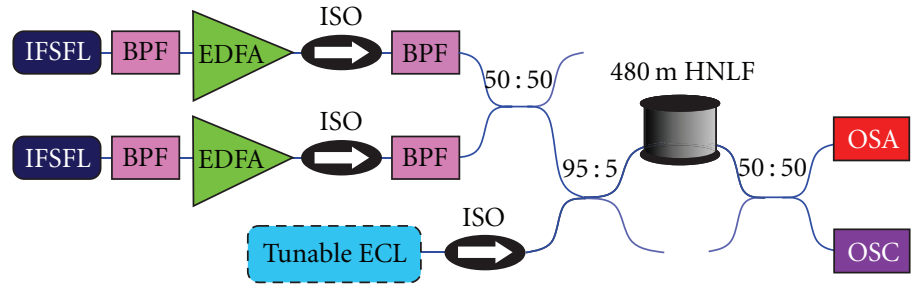

Figure 1: Experimental setup; IFSFL: intra-cavity frequency-shifted feedback laser, BPF: bandpass filter, EDFA: erbium-doped fiber amplifier, ISO: isolator, ECL: extended cavity tunable laser, HNLF: highly non-linear fiber, OSA: optical spectrum analyzer, and OSC: oscilloscope.

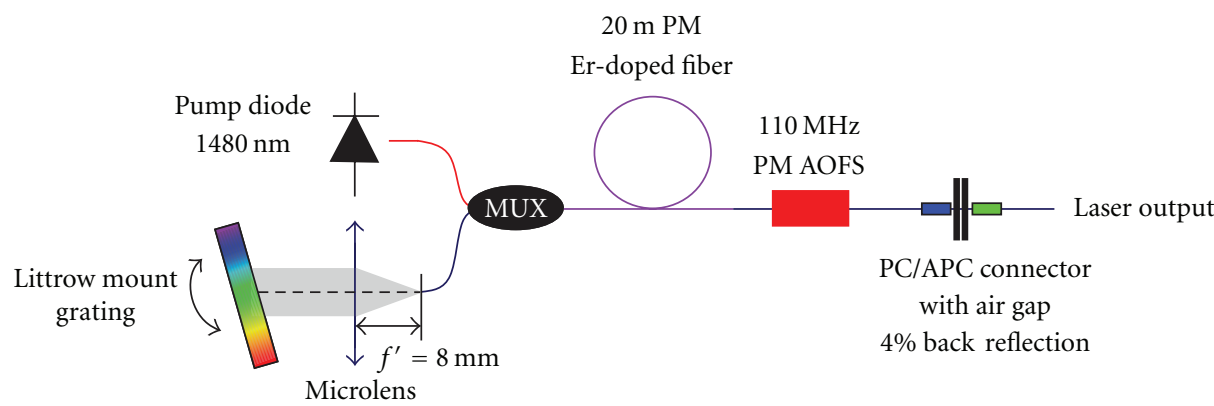

FIGURE 2: Setup of each IFSFL; MUX: multiplexer, PM: polarization maintaining, and AOFS: acousto-optic frequency shifter.

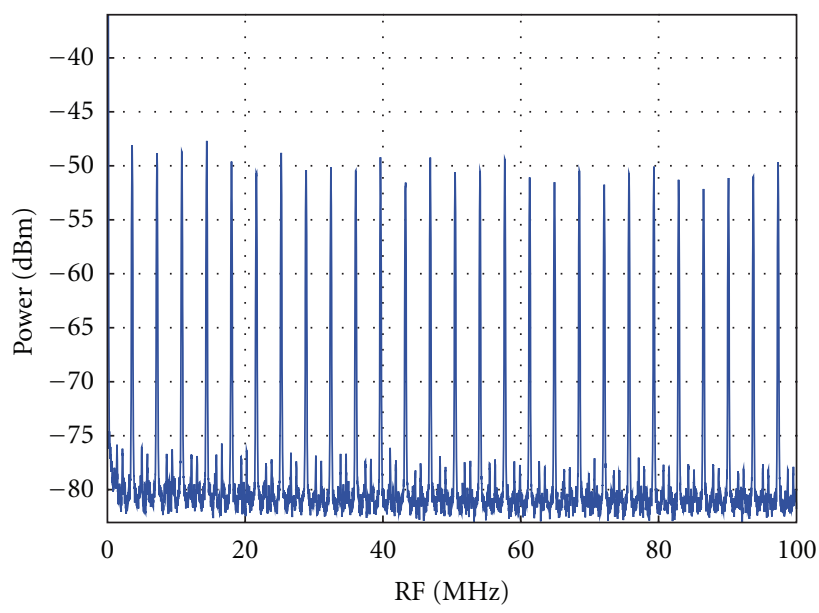

(a)

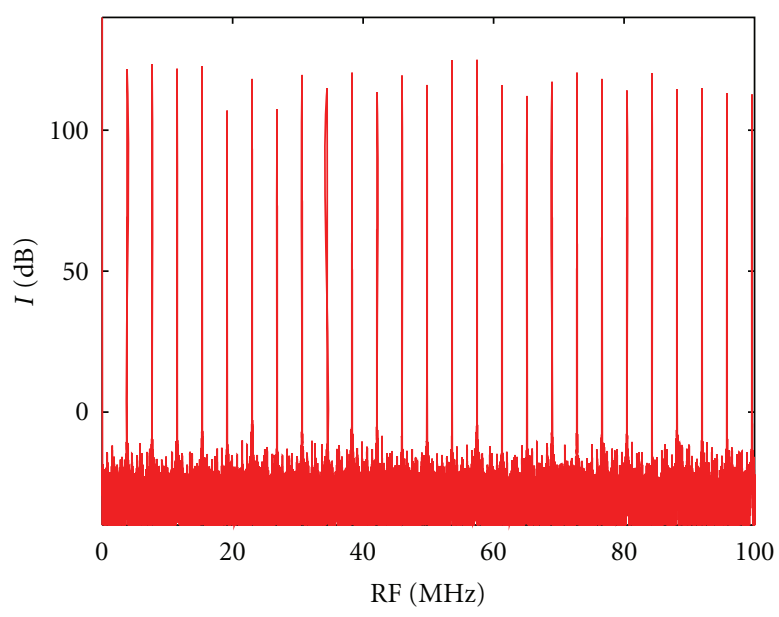

(b)

FIGURE 3: (a) Experimental RF spectrum for IFSFL 1 with a preamplified RF analyzer: the frequency comb free spectral range is of $3.6 \mathrm{MHz}$; (b) numerical simulation of RF photodiode current (spectrum of equation (3) of [17]).

between the pumps may drastically degrade the conversion efficiency in a dual pump process. According to [18], the bifurcation point between CW and pulsed solution can be influenced by the pump diode power. To keep the IFSFLs operating in the CW regime, we have then set their diode currents to a low value (65 $\mathrm{mA}$ for both lasers). For higher values of diode current one observes a rapid transition to time-periodic solutions [18] by direct observation of the laser output through a photodiode and an oscilloscope. The working points of our lasers were finely adjusted by verifying the absence of relaxation oscillations with an oscilloscope and a $1 \mathrm{GHz}$ bandwidth InGaAs photodetector.

\section{Background Theory}

Four-wave mixing is usually characterized as 3 distinct processes depending on the relative positions of the pumps, signal, and idler frequencies (see Figure 4(a)) $[4,8]$ : modulation instability (MI), phase conjugation (PC), and Bragg-scattering (BS). All of them may be simultaneously 


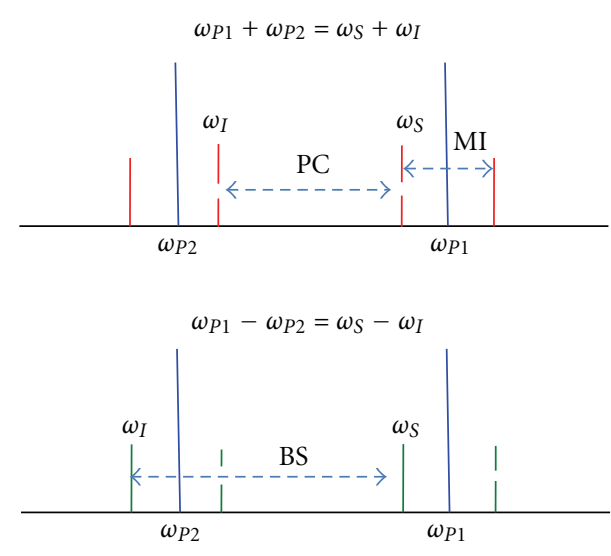

(a)

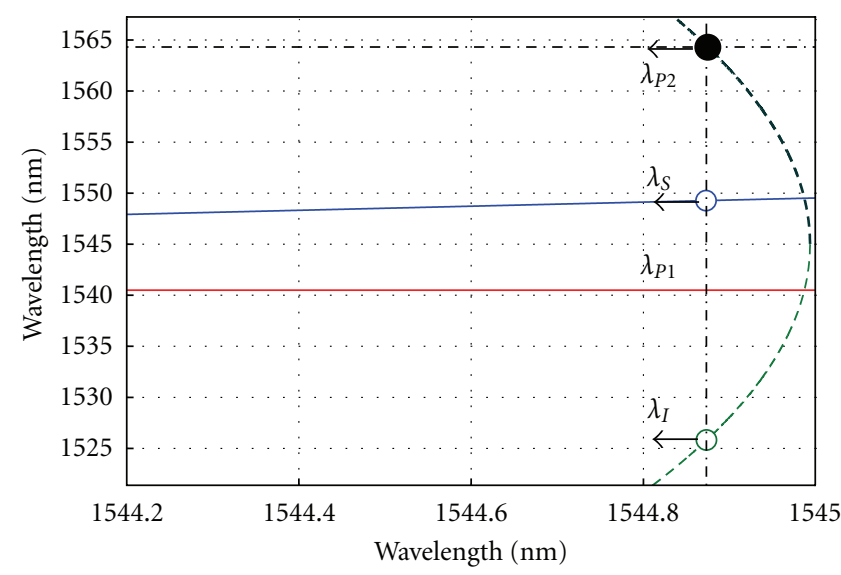

(b)

Figure 4: (a) Four-wave mixing processes, from [4]; (b) example of BS-FWM phase-matching diagram: thick solid (dashed) line (curve) for pump 1 (pump 2); thin solid (dashed) line (curve) for signal (idler).

observable [5] especially for low dispersion values, as it will be shown at the end of Section 4. MI requires one pump process. PC and BS require instead two distinct pumps. BSFWM has a low noise nature as the frequency conversion may take place at phase matching in the absence of exponential gain.

For BS-FWM energy and momentum conservation give for angular frequencies $\omega_{j}$ and wave-vector $k_{j}(j=P 1, P 2, S$, for the pump 1, pump 2 , signal and idler resp.) $[4,5,11]$ :

$$
\begin{gathered}
\omega_{I}=\omega_{S}+\left(\omega_{P 1}-\omega_{P 2}\right), \\
k_{I}=k_{S}+\left(k_{P 1}-k_{P 2}\right) .
\end{gathered}
$$

The dependence of wave vectors on angular frequency leads to the determination of the best conditions for frequency conversion. In close proximity to the ZDW, the dispersion coefficient of an HNLF is generally approximated by a linear function of wavelength. Under this approximation, if one fixes for instance the wavelength of the pump 1 , one can build a chart of optimization for the remaining wavelengths combining (1) and (2) $[4,5]$. An example for such a diagram is given in Figure 4(b) for a fiber with ZDW of $1545 \mathrm{~nm}$ and dispersion slope of $0.018 \mathrm{ps} /\left(\mathrm{km} \mathrm{nm}^{2}\right)$. The horizontal axis represents a common reference wavelength: its corresponding angular frequency is the mean value between the frequency of the idler and that of pump 2. One can start to choose a value for the wavelength for pump 2 by covering the corresponding thick dashed curve: the wavelength for pump 2 will be the vertical coordinate of the selected point. Then from the chart one can automatically predict the best signal wavelength and hence the position of the corresponding idler. One should draw first a vertical line that matches the selected choice of pump 2. The wavelengths for signal and idler will be read at the intersection points with the other curves. More specifically the signal will be read at the intersection with the thin solid line while the idler will come from the intersection with the thin dashed curve. In practice, it is likely that the ZDW may fluctuate by about $1 \mathrm{~nm}$ along the fiber length, according to the analysis presented in [19] for similar types of fibers. This fact may degrade the overall conversion efficiency [5].

\section{Experimental Results and Discussion}

Let us first focus on the spectral region between $1520 \mathrm{~nm}$ and $1535 \mathrm{~nm}$, where the BS-FWM appears whenever the signal is varied between $1547.3 \mathrm{~nm}$ and $1554.3 \mathrm{~nm}$. The collection of these experimental results is shown in Figure 5(a). Note that the BS-FWM sideband varies from $1524.5 \mathrm{~nm}$ to $1531.5 \mathrm{~nm}$ and keeps a fixed distance of $2.87 \mathrm{THz}$ from the signal. BSFWM is then a follower of the signal frequency, being the frequency distance between signal and idler in BS-FWM decided by the pumps frequencies. We better show this effect in Figure 5(b) where the experimental results of signal and idler carrier wavelength compare well with the energy conservation law of (1). However, in the same spectral range we observe a competition with the degenerated FWM driven by pump 1 . Hereafter we will indicate this effect as modulation instability (MI) independently from the presence or absence of parametric gain. Note that we work with very low dispersion values and one of these sidebands is seeded by the input signal itself. Differently from BS-FWM, the sidebands of MI-FWM follow a symmetry law around the pump. Such symmetry in spectrum is very well known and can be understood again from the energy conservation law that reads as $\omega_{I}=2 \omega_{P 1}-\omega_{S}$. If we assume that the angular frequency of signal is detuned from that of a pump $P 1$ as $\omega_{S}=\omega_{P 1}-\Omega$, then it turns out that $\omega_{I}=\omega_{P 1}+\Omega$.

Therefore if we keep fixed the spectral position of pumps and we gradually reduce the angular frequency $\omega_{S}$ (i.e., we increase the signal wavelength) we can observe two opposite behaviors: the angular frequency $\omega_{I}$ of degenerated FWM will increase whereas that of BS-FWM will decrease. In our experiment this fact leads even to a superposition of the two idlers at $1528.75 \mathrm{~nm}$, when the signal is tuned precisely at $\lambda_{S}=1551.42 \mathrm{~nm}$. This crossing effect upon signal wavelength 


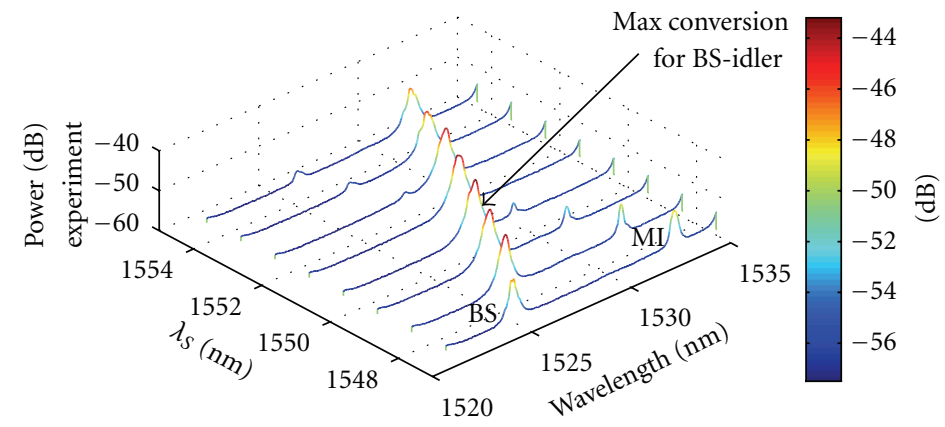

(a)

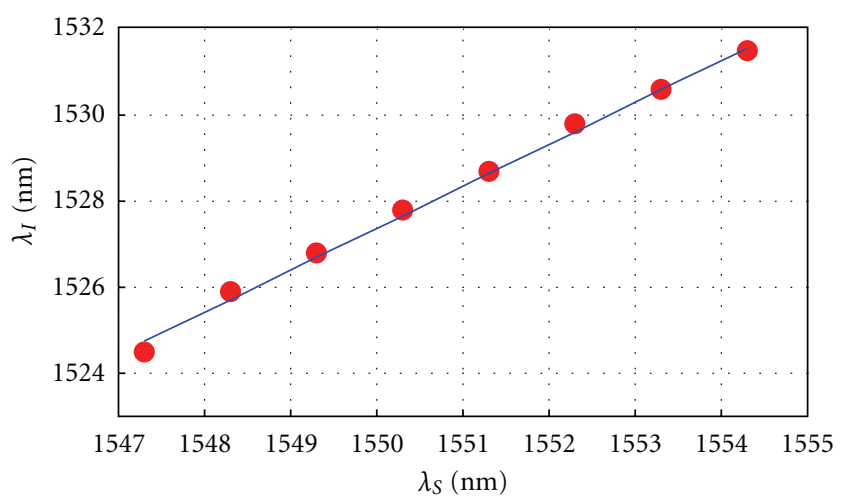

(b)

FIGURE 5: (a) Collection of experimental spectra upon different input signal wavelengths; (b) BS-FWM idler wavelength upon signal wavelength; dots: experimental results; solid line: equation (1).

is also shown in Figure 5(a). The coexistence of these two parametric processes and their tiny frequency separation should be then considered carefully in applications. Note that pump 1 is very close to the ZDW and a selection rule of parametric processes, based on phase mismatch may become less effective in practice.

As we show in Figure 6(a), we obtained in our experiment $16 \mathrm{~dB}$ from the background noise level at the idler central wavelength for the case of maximally efficient BSFWM. Note that in our spectral measurements we set to $1 \mathrm{kHz}$ the video bandwidth digital filter of the optical spectrum analyzer. The maximum conversion is found for a signal at $1550.3 \mathrm{~nm}$. The discrepancy from phase matching $(1549.8 \mathrm{~nm})$ may be due to uncertainties in the dispersion parameters of the fiber and to the fluctuation in ZDW along the beam propagation in the fiber [5], as we have already mentioned. We obtained a depletion of the signal of about $1 \mathrm{~dB}$ and a ratio between the spectral peak of the idler and that of the signal of the signal of $-20 \mathrm{~dB}$ : this result is much lower than that reported in [5] for the case of coherent pumps with direct phase modulation. This is mainly due to the difference in fiber parameters and choice of wavelengths. Additionally note that the experiments show that the idler is spectrally broadened as it comes from a combination of broadband pumps and a narrowband signal [6]. In the experiments we estimated the $3 \mathrm{~dB}$ bandwidths of pumps as $20 \mathrm{GHz}$ and $44 \mathrm{GHz}$ for IFSL 1 and 2, respectively, (our spectrum analyzer has a minimum resolution of $0.05 \mathrm{~nm}$ which correspond to $6.24 \mathrm{GHz}$ at these wavelengths). We experimentally obtained a $57 \mathrm{GHz}$ wide BS-idler at $1527.8 \mathrm{~nm}$ and for a signal wavelength $\lambda_{S}=$ $1550.3 \mathrm{~nm}$.

Note that in our experiment we can shift the signal within the relatively wide spectral range and still obtain efficient BS-idler generation (see Figure 5(b)). This effect may be potentially applicable for the simultaneous conversion of a large spectrum. In our case, the conversion efficiency BSFWM varies by less than $3 \mathrm{~dB}$ when the signal is tuned by $9 \mathrm{~nm}$.

Let us now report on the case of the two external sidebands (see $a$ and $b$ in Figure 6(b)). By using the word "spontaneous" in the label, we mean that these sidebands are directly generated by the two pumps and they do not require a third signal to appear. Their spectral position is entirely due to the spectral positions of pumps: with respect to pump 1 (pump 2), sideband $a$ (b) is at the same frequency detuning of pump 2 (pump 1). Because of the low dispersion of the fiber and relatively high intensity of pumps, sidebands $a$ and $b$ have large amplitudes. Note that the amplitude of the left-side spontaneous FWM idler (see $a$ in Figure 6(b)) is larger than that of the right-side one (see $b$ in Figure 6(b)) There are also some additional frequency conversion effects. Since their appearance is strongly connected to the fine adjustment of the working points of IFSFLs, we report our best observation in Figure 7. More specifically we observed the signature of MI-FWM around pump 2 and PC-FWM. 


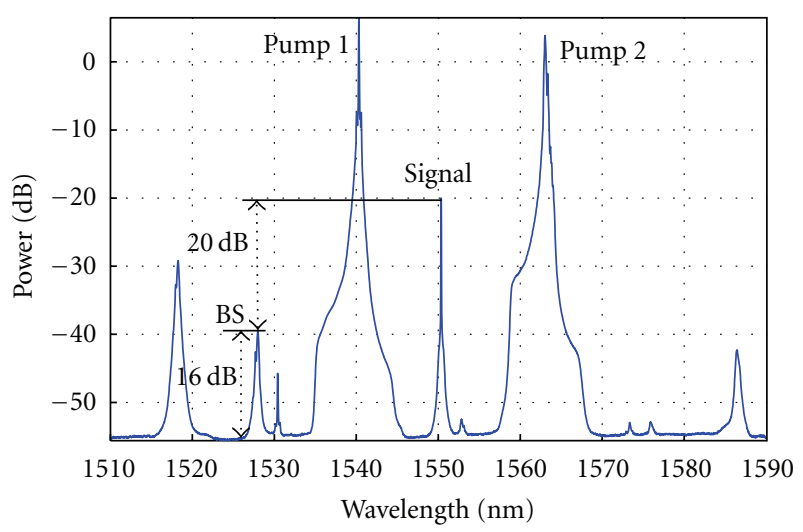

(a)

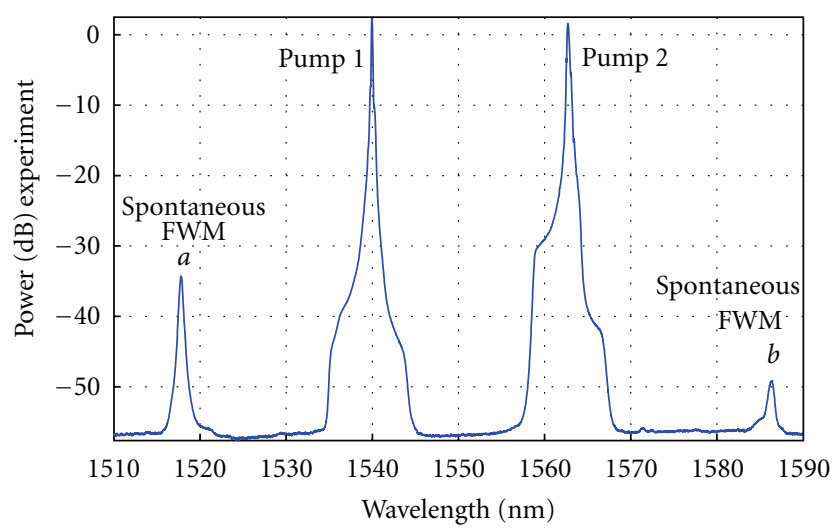

(b)

FIGURE 6: (a) experimental output spectrum obtained for the signal wavelength at $1550.3 \mathrm{~nm}$ : maximal conversion of BS-FWM; phasematching conditions for BS-FWM. (b) experimental output with two pumps only.

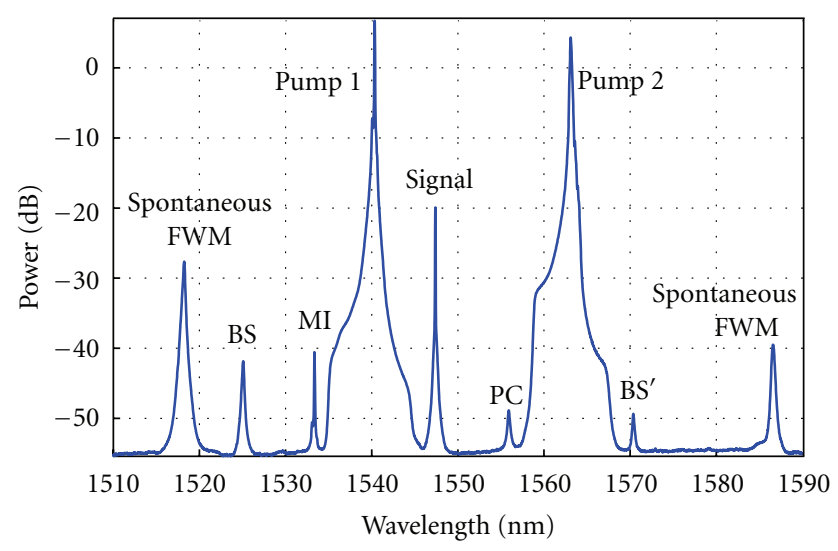

(a)

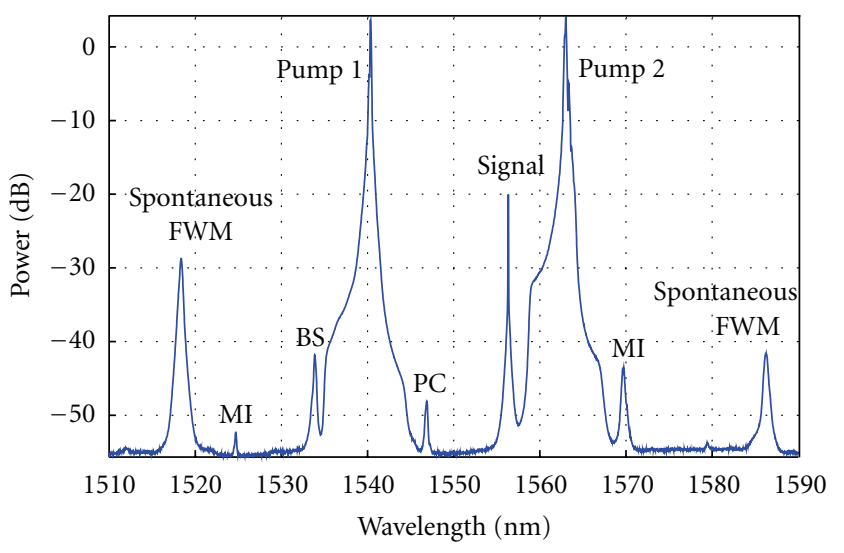

(b)

Figure 7: Experimental output spectrum obtained for signal wavelength at $1547.3 \mathrm{~nm}$ (a) and $1556.3 \mathrm{~nm}$ (b); existence of multiple simultaneous FWM processes.

We also observed another kind of BS-FWM that comes from the subtraction instead of the sum of the beating frequency of $2.87 \mathrm{THz}\left(\mathrm{BS}^{\prime}\right)$. The conversion efficiencies of these last processes remain low, probably due to the large phase mismatch. We show in Figure 7 two output spectra taken with a signal at $1547.3 \mathrm{~nm}$ (a) and $1556.3 \mathrm{~nm}$ (b).

\section{Conclusion}

In this paper, we studied BS-FWM in HNLF with a novel cost-effective and partially coherent pumping scheme. We experimentally demonstrated efficient and tunable BS-FWM despite the large bandwidth of the pumps and their complete statistical independence. The spectral peak of the converted BS-FWM is $20 \mathrm{~dB}$ below that of the original signal and $16 \mathrm{~dB}$ above the background noise level. We noted coexistence of multiple independent FWM processes. In particular, we observed a relevant competition BS-FWM and MI-FWM. Our measurements agree well with a theoretical analysis based on the phase-matching diagram for the case of monochromatic waves. In conclusion, nonlinear fiber optics frequency converters based on broadband pumps appear to be promising for their implementation in all-optical signal processing devices.

\section{Acknowledgments}

The authors acknowledge the financial support from the Region Limousin and from the French National Research Agency under Grant ANR-08-JCJC-0122 PARADHOQS. They thank Alain Dexet for his assistance in manufacturing some of the mechanical parts used in the experiment.

\section{References}

[1] C. J. McKinstrie, S. Radic, and A. R. Chraplyvy, "Parametric amplifiers driven by two pump waves," IEEE Journal on Selected Topics in Quantum Electronics, vol. 8, no. 3, pp. 538547, 2002.

[2] R. Slavík, F. Parmigiani, J. Kakande et al., "All-optical phase and amplitude regenerator for next-generation telecommunications systems," Nature Photonics, vol. 4, no. 10, pp. 690-695, 2010 . 
[3] J. Hansryd, P. A. Andrekson, M. Westlund, J. Li, and P. O. Hedekvist, "Fiber-based optical parametric amplifiers and their applications," IEEE Journal on Selected Topics in Quantum Electronics, vol. 8, no. 3, pp. 506-520, 2002.

[4] D. Méchin, R. Provo, J. D. Harvey, and C. J. McKinstrie, "180$\mathrm{nm}$ wavelength conversion based on Bragg scattering in an optical fiber," Optics Express, vol. 14, no. 20, pp. 8995-8999, 2006.

[5] R. Provo, S. Murdoch, J. D. Harvey, and D. Méchin, "Bragg scattering in a positive $\beta_{4}$ fiber," Optics Letters, vol. 35 , no. 22 , pp. 3730-3732, 2010.

[6] Y. Yan and C. Yang, "Four-wave mixing between coherent signal and incoherent pump light in nonlinear fiber," Journal of Lightwave Technology, vol. 27, no. 22, pp. 4954-4959, 2009.

[7] D. Nodop, D. Schimpf, J. Limpert, and A. Tünnermann, "SBS suppression in high power fiber pulse amplifiers employing a superluminescence diode as seed source," in Proceedings of the Conference on Lasers and Electro-Optics (CLEO EUROPE '11), Munich, Germany, May 2011, paper CJ7.6 THU.

[8] A. H. Gnauck, R. M. Jopson, C. J. McKinstrie, J. C. Centanni, and S. Radic, "Demonstration of low-noise frequency conversion by Bragg Scattering in a Fiber," Optics Express, vol. 14, no. 20, pp. 8989-8994, 2006.

[9] S. Radic, R. M. Jopson, A. Gnauck, C. J. McKinstrie, J. C. Centanni, and A. R. Chaplyvy, "Stimulated-brillouinscattering supression using a single modulator in two-pump parametric architectures," in Proceedings of the Optical Fiber Communication Conference (OFC '05), Anaheim, Calif, USA, March 2005, paper OWN5.

[10] J. Schröder, A. Boucon, S. Coen, and T. Sylvestre, "Interplay of four-wave mixing processes with a mixed coherent-incoherent pump," Optics Express, vol. 18, no. 25, pp. 25833-25838, 2010.

[11] H. J. McGuinness, M. G. Raymer, C. J. McKinstrie, and S. Radic, "Quantum frequency translation of single-photon states in a photonic crystal fiber," Physical Review Letters, vol. 105, no. 11, Article ID 093604, 2010.

[12] Y. Yan and Y. Changxi, "Coherent light wave generated from incoherent pump light in nonlinear kerr medium," Journal of the Optical Society of America B, vol. 26, no. 11, pp. 2059-2063, 2009.

[13] H. G. Chatellus and J.-P. Pique, "Coherence properties of modeless lasers," in Proceedings of the Science, Quantum of Quasars Workshop, Grenoble, France, December 2009.

[14] L. P. Yatsenko, B. W. Shore, and K. Bergmann, "Coherence in the output spectrum of frequency shifted feedback lasers," Optics Communications, vol. 282, no. 2, pp. 300-309, 2009.

[15] L. P. Yatsenko, B. W. Shore, and K. Bergmann, "An intuitive picture of the physics underlying optical ranging using frequency shifted feedback lasers seeded by a phase-modulated field," Optics Communications, vol. 282, no. 11, pp. 2212-2216, 2009.

[16] P. A. Champert, V. Couderc, and A. Barthélémy, "1.5-2.0- $\mu \mathrm{m}$ multiwatt continuum generation in dispersion-shifted fiber by use of high-power continuous-wave fiber source," IEEE Photonics Technology Letters, vol. 16, no. 11, pp. 2445-2447, 2004.

[17] K. Nakamura, T. Miyahara, and H. Ito, "Observation of a highly phase-correlated chirped frequency comb output from a frequency-shifted feedback laser," Applied Physics Letters, vol. 72, no. 21, pp. 2631-2633, 1998.

[18] M. Stellpflug, G. Bonnet, B. W. Shore, and K. Bergmann, "Dynamics of frequency shifted feedback lasers: Simulation studies," Optics Express, vol. 11, no. 17, pp. 2060-2080, 2003.
[19] B. Auguié, A. Mussot, A. Boucon, E. Lantz, and T. Sylvestre, "Ultralow chromatic dispersion measurement of optical fibers with a tunable fiber laser," IEEE Photonics Technology Letters, vol. 18, no. 17, pp. 1825-1827, 2006. 

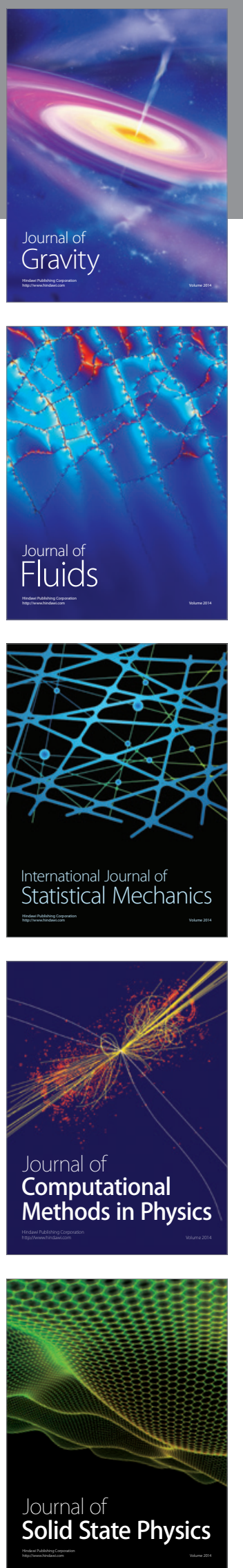

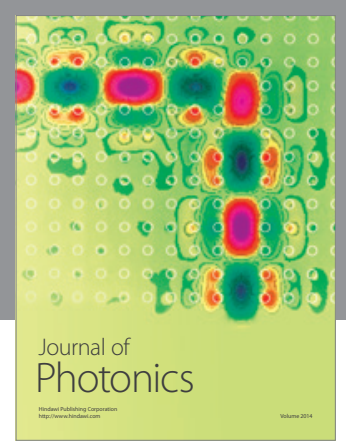

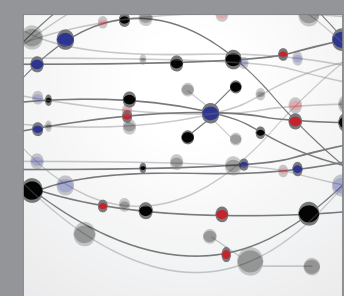

The Scientific World Journal
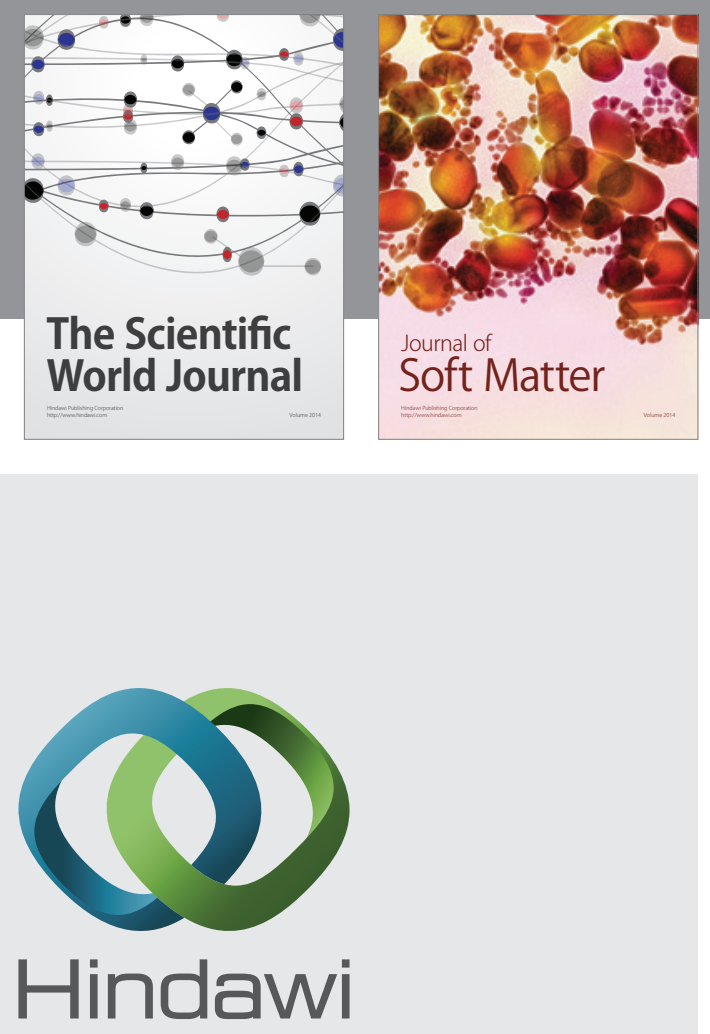

Submit your manuscripts at

http://www.hindawi.com
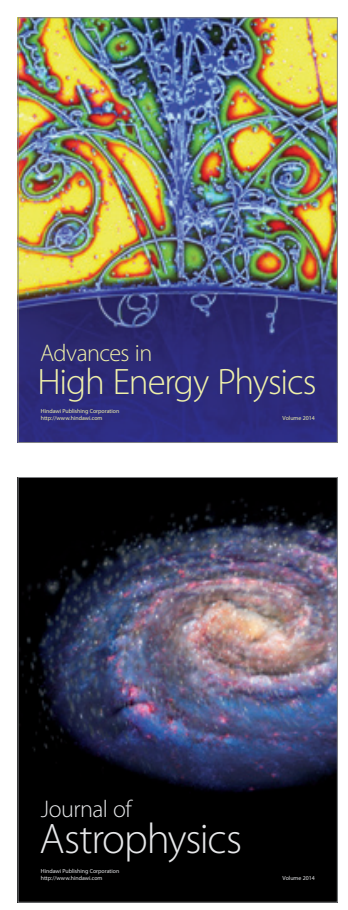
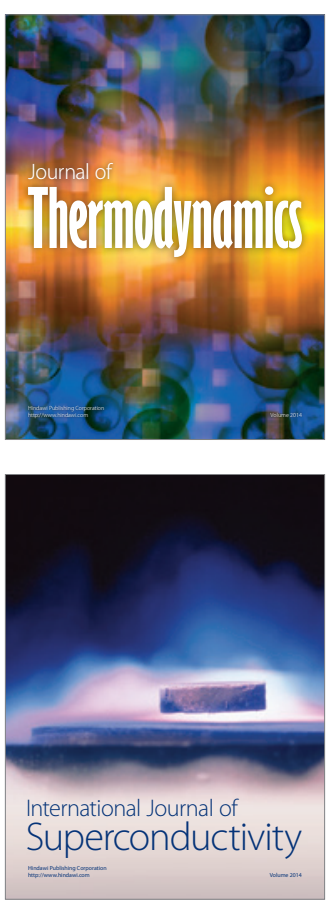
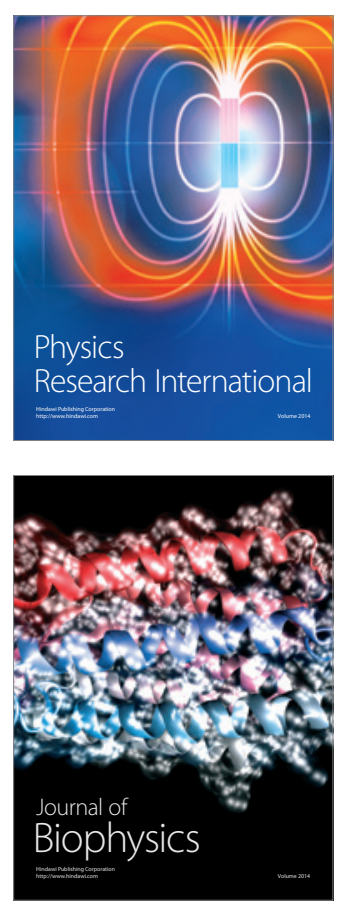
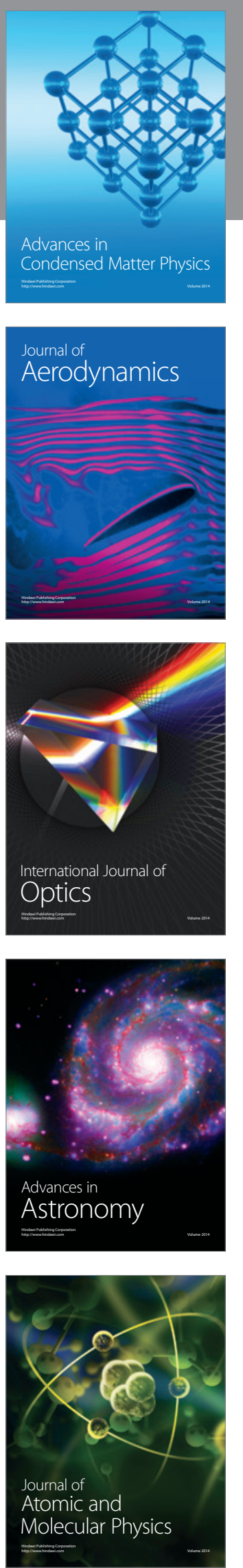